\title{
TEM Characterization of Textured Alumina Produced by Templated Grain Growth (TGG)
}

\section{B.J. Hockey* and G.L. Messing**}

*Ceramics Division, National Institute of Standards and Technology, Gaithersburg, MD 20899

** The Pennsylvania State University, University Park, PA 16802

Current interest in polycrystalline ceramics having properties that reflect anisotopic characteristics inherent to their single crystal forms has led to development of new processing techniques for producing textured microstructures. One such technique is Templated Grain Growth (TGG), which has been developed and used successfully by Messing and coworkers to produce highly textured bodies of alumina and other materials.[1] For alumina, their processing scheme and the evolution of textured microstructures have been detailed elsewhere.[e.g. 1,2] For brevity, we note only that the starting components consisted of [0001], c-axis, oriented $\alpha-\mathrm{Al}_{2} \mathrm{O}_{3}$ platelets (approx. $11 \mu \mathrm{m}$ in dia. and $1.5 \mu \mathrm{m}$ in thickness), which were aligned within a "matrix" containing a sol-gel derived alumina precursor seeded with nanosized $\alpha$-phase powder and a silica/calcia glass-forming precursor (to promote liquid phase sintering). In the present study, which is designed to provide a more detailed description of the microstructure, the samples were sintered at $1600^{\circ} \mathrm{C}$ for $2 \mathrm{~h}$ and contained 5 v/o platelets with either 1 or 10 v/o calcia-alumino silicate glass phase.

In general, TEM examination confirmed the textured nature of the microstructure relates to the rapid growth of the $\alpha-\mathrm{Al}_{2} \mathrm{O}_{3}$ platelets into a reasonably aligned configuration of similarly shaped, large grains, having "diameters" of 30-50 $\mu \mathrm{m}$ and thicknesses roughly one-tenth the diameter. Figure 1a illustrates the aligned morpholgy of large grains in crosssection, while Figure $2 \mathrm{a}$ illustrates the irregular shape of a large c-axis oriented grain in plan-view (here, tilted off axis). Our observations also revealed that many of these large, plateletshaped grains are twinned, or more precisely, contain segments that are related by a basal twin operation (i.e., a $180^{\circ}$ rotation about the [0001], c-axis for rhombohedrally symmetric $\alpha-\mathrm{Al}_{2} \mathrm{O}_{3}$ ), Figure $1 \mathrm{~b}$. Moreover, as seen in the cross-sectional views, Figures $1 \mathrm{a}$ and $\mathrm{b}$, the twin boundaries are not parallel to the basal planes, but instead are steeply inclined to (0001) and are more aptly called twin walls. In the plan-views, Figures $2 \mathrm{a}$ and, in more detail, Fig. $2 \mathrm{~b}$, the meandering morpholgy of the twin walls is seen to produce a complex, highly irregular twinned substructure. Consideration of the origin of this unusual twinned grain structure led to examination of the initial platelet particles, Figure 3. Based on an admittedly small sampling (12), only a small fraction of platelets (1/6) were found to contain a similar twinned substructure, e.g. 3a; most were not twinned, Figure 3b.

Thus, while the propagation of twinned segments from some of the template platelets will occur during grain growth, the possibility that other processes contribute to the formation of the observed twinned substructure cannot be discounted. In particular, consideration of the abrupt change in the cross-sectional profile of the twinned grain seen in Fig. 1b suggests that the radial growth may involve the joining of similarly oriented or twin oriented grains.

References

[1] E. Suvaci, K.-S, Oh and G.L. Messing, Acta mater. 49, 2075-2081 (2001)

[2] M.M. Seabaugh, I.H. Kerscht and G.L. Messing, J. Am. Ceram. Soc., 80, 118188 (1997) 

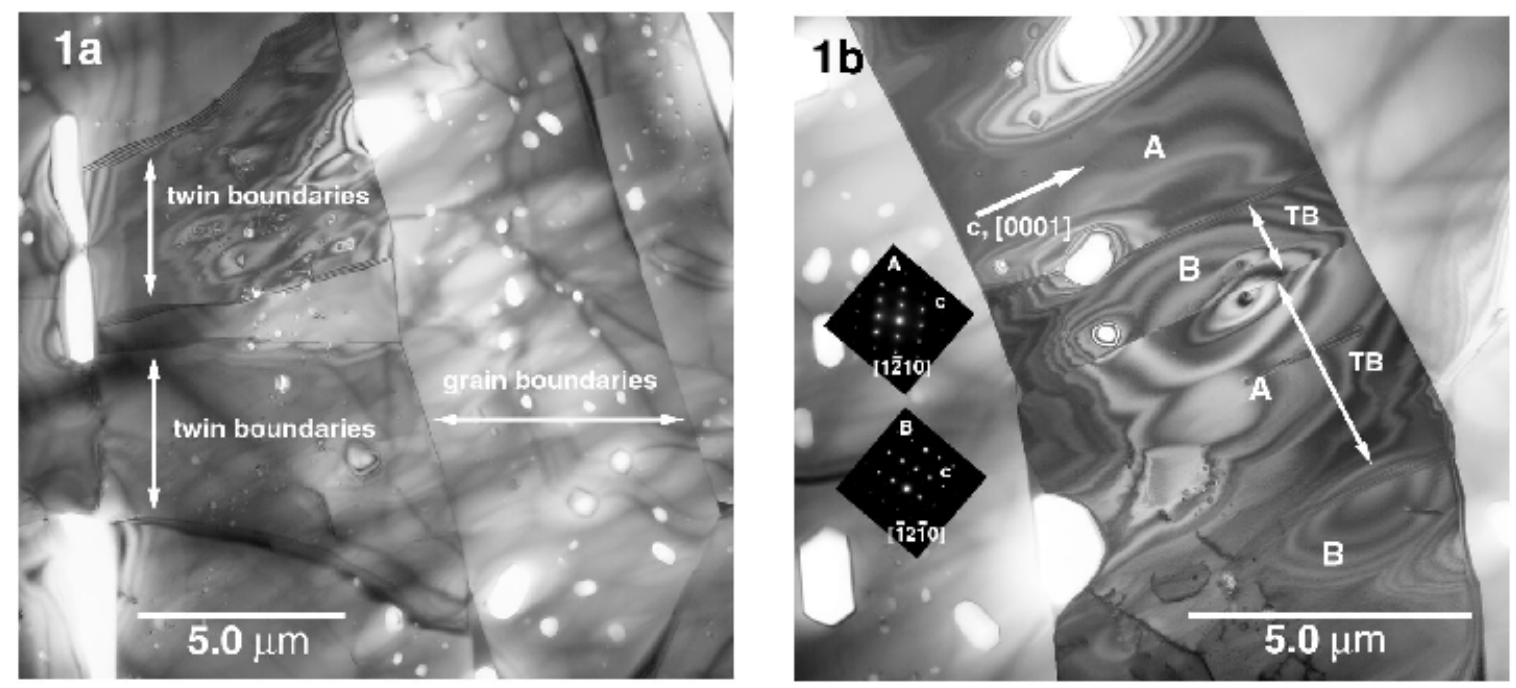

Figure 1 Cross-section views of large plate-shaped grains in TGG sintered material. Grains are subdivided into segments which are basal twin related.
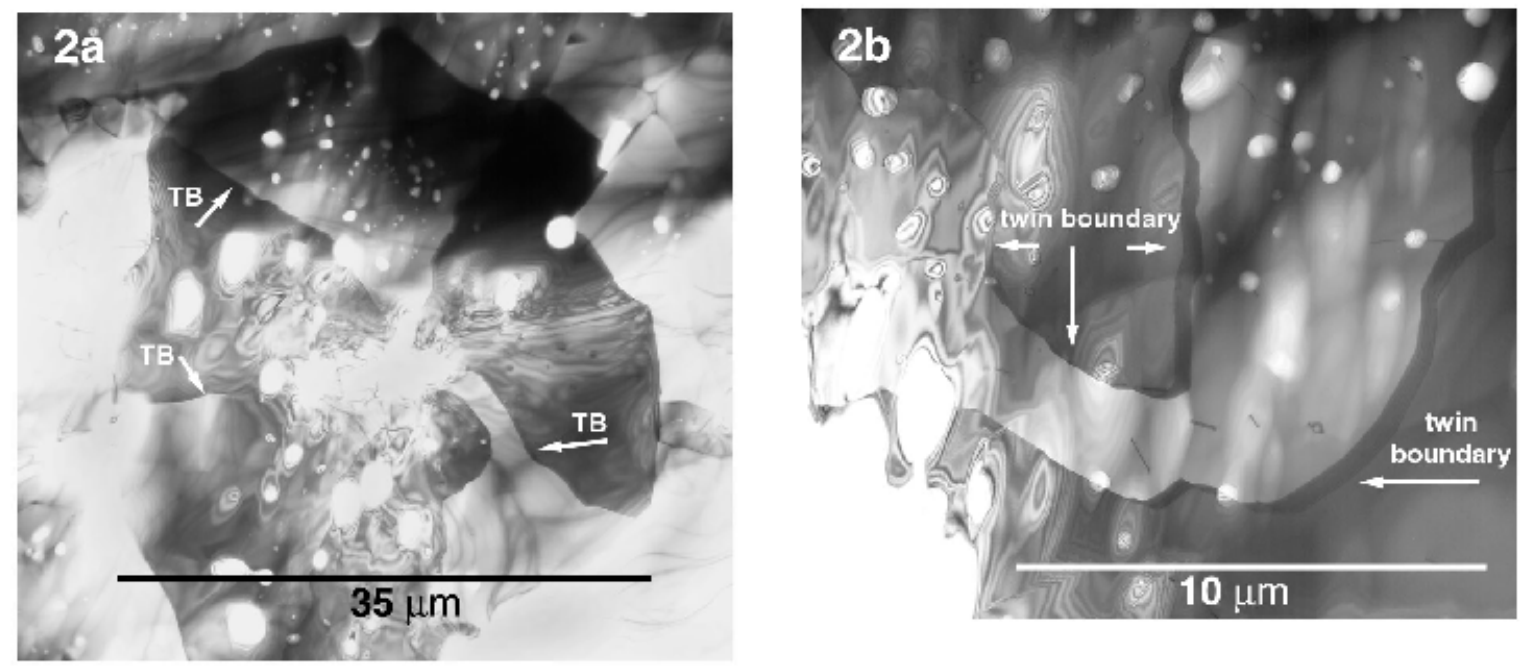

Figure 2 Plan views of large plate-shaped grains in sintered material.

a) note irregular boundary and twinned substructure with twin boundaries (TB)

b) illustrates irregular morphology of twin boundaries
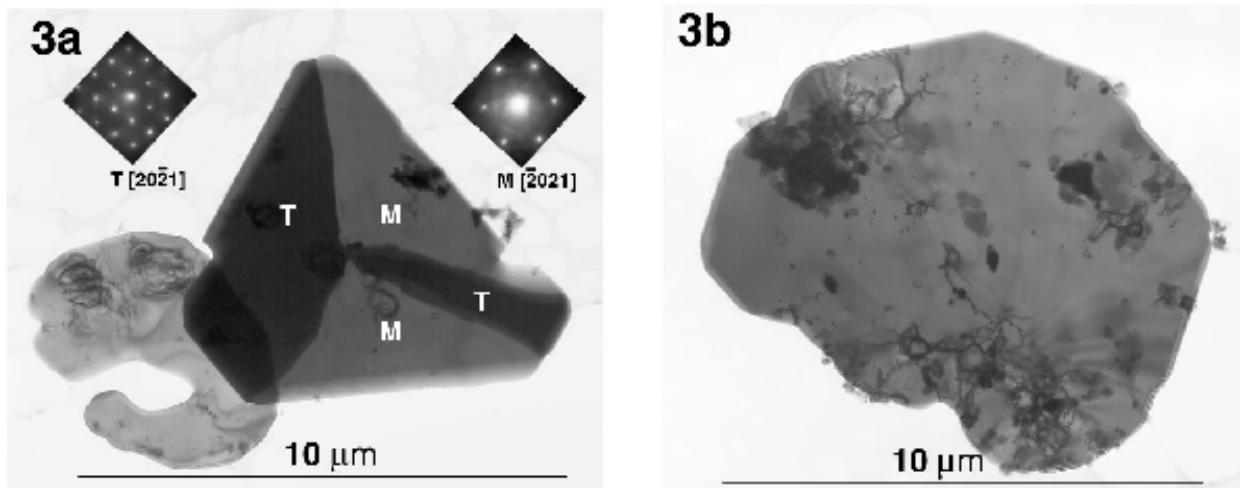

Figure 3 Twinned (a) and untwinned (b) template particles 